\title{
ON THE FORMAL GROUP LAWS OF UNORIENTED AND COMPLEX COBORDISM THEORY
}

\author{
BY DANIEL QUILLEN ${ }^{1}$ \\ Communicated by Frank Peterson, May 16, 1969
}

In this note we outline a connection between the generalized cohomology theories of unoriented cobordism and (weakly-) complex cobordism and the theory of formal commutative groups of one variable [4], [5]. This connection allows us to apply Cartier's theory of typical group laws to obtain an explicit decomposition of complex cobordism theory localized at a prime $p$ into a sum of Brown-Peterson cohomology theories [1] and to determine the algebra of cohomology operations in the latter theory.

1. Formal group laws. If $R$ is a commutative ring with unit, then by a formal (commutative) group law over $R$ one means a power series $F(X, Y)$ with coefficients in $R$ such that

(i) $F(X, 0)=F(0, X)=X$,

(ii) $F(F(X, Y), Z)=F(X, F(Y, Z))$,

(iii) $F(X, Y)=F(Y, X)$. We let $I(X)$ be the "inverse" series satisfying $F(X, I(X))=0$ and let

$$
\omega(X)=d X / F_{2}(X, 0)
$$

be the normalized invariant differential form, where the subscript 2 denotes differentiation with respect to the second variable. Over $R \otimes Q$, there is a unique power series $l(X)$ with leading term $X$ such that

$$
l(F(X, Y))=l(X)+l(Y) .
$$

The series $l(X)$ is called the logarithm of $F$ and is determined by the equations

$$
\begin{aligned}
l^{\prime}(X) d X & =\omega(X), \\
l(0) & =0 .
\end{aligned}
$$

2. The formal group law of complex cobordism theory. By complex cobordism theory $\Omega^{*}(X)$ we mean the generalized cohomology theory associated to the spectrum $M U$. If $E$ is a complex vector bundle of dimension $n$ over a space $X$, we let $c_{i}^{\Omega}(E) \in \Omega^{2 i}(X), 1 \leqq i \leqq n$ be

${ }^{1}$ Alfred P. Sloan Foundation Fellow. This work was supported also by NSF GP9006. 
the Chern classes of $E$ in the sense of Conner-Floyd [3]. Since $\Omega^{*}\left(C P^{\infty} \times C P^{\infty}\right)=\Omega^{*}(p t)[[x, y]]$, where $x=c_{1}^{\Omega}(\mathcal{O}(1)) \otimes 1, y=1 \otimes c_{1}^{\Omega}(\mathcal{O}(1))$ and $\mathcal{O}(1)$ is the canonical line bundle on $C P^{\infty}$, there is a unique power series $F^{\mathfrak{a}}(X, Y)=\sum a_{k l} X^{k} Y^{l}$ with $a_{k l} \in \Omega^{2-2 k-2 l}(p t)$ such that

$$
\stackrel{\Omega}{c_{1}}\left(L_{1} \otimes L_{2}\right)=F^{\Omega}\left(c_{1}^{\Omega}\left(L_{1}\right), \stackrel{\Omega}{c_{1}}\left(L_{2}\right)\right)
$$

for any two complex line bundles with the same base. The power series $F^{2}$ is a formal group law over $\Omega^{e v}(p t)$.

THEOREM 1. Let $E$ be a complex vector bundle of dimension $n$, let $f: P E^{\prime} \rightarrow X$ be the associated projective bundle of lines in the dual $E^{\prime}$ of $E$, and let $O(1)$ be the canonical quotient line bundle on $P E^{\prime}$. Then the Gysin homomorphism $f_{*}: \Omega^{q}\left(P E^{\prime}\right) \rightarrow \Omega^{q-2 n+2}(X)$ is given by the formula

$$
f_{*}(u(\xi))=\operatorname{res} \frac{u(Z) \omega(Z)}{\prod_{j=1}^{n} F^{\mathbb{Q}}\left(Z, I \lambda_{j}\right)} .
$$

Here $u(Z) \in \Omega(X)[Z], \xi=c_{1}^{\Omega}(\mathcal{O}(1)), \omega$ and I are the invariant differential form and inverse respectively for the group law $F^{2}$, and the $\lambda_{j}$ are the dummy variables of which $c_{q}^{\Omega}(E)$ is the qth-elementary symmetric function.

The hardest part of this theorem is to define the residue; we specialize to dimension one an unpublished definition of Cartier, which has also been used in a related form by Tate [7].

Applying the theorem to the map $f: C P^{n} \rightarrow p t$, we find that the coefficient of $X^{n} d X$ in $\omega(X)$ is $P_{n}$, the cobordism class of $C P^{n}$ in $\Omega^{-2 n}(p t)$. From (2) we obtain the

CoROLlary (MYSHENKo [6]). The logarithm of the formal group law of complex cobordism theory is

$$
l(X)=\sum_{n \geq 0} P_{n} \frac{X^{n+1}}{n+1}
$$

\section{The universal nature of cobordism group laws.}

THEOREM 2. The group law $F^{\Omega}$ over $\Omega^{e v}(p t)$ is a universal formal (commutative) group law in the sense that given any such law $F$ over a commutative ring $R$ there is a unique homomorphism $\Omega^{e v}(p t) \rightarrow R$ carrying $F^{2}$ to $F$.

Proof. Let $F_{u}$ over $L$ be a universal formal group law [5] and let $h: L \rightarrow \Omega^{e v}(p t)$ be the unique ring homomorphism sending $F_{u}$ to $F^{\Omega}$. The law $F_{u}$ over $L \otimes Q$ is universal for laws over $\boldsymbol{Q}$-algebras. Such a 
law is determined by its logarithm series which can be any series with leading term $X$. Thus if $\sum p_{n} X^{n+1} / n+1$ is the logarithm of $F_{u}, L \otimes Q$ is a polynomial ring over $Q$ with generators $p_{i}$. By (5) $h p_{i}=P_{i}$, so as $\Omega^{*}(p t) \otimes Q \cong Q\left[P_{1}, P_{2}, \cdots\right]$, it follows that $h \otimes Q$ is an isomorphism.

By Lazard [5, Theorem II], $L$ is a polynomial ring over $\boldsymbol{Z}$ with infinitely many generators; in particular $L$ is torsion-free and hence $h$ is injective. To prove surjectivity we show $h(L)$ contains generators for $\Omega^{*}(p t)$. First of all $h p_{n}=P_{n} \in h(L)$ because $p_{n} \in L$ as it is the $n$th coefficient of the invariant differential of $F_{u}$. Secondly we must consider elements of the form $\left[M_{n}\right]$ where $M_{n}$ is a nonsingular hypersurface of degree $k_{1}, \cdots, k_{r}$ in $C P^{n_{1}} \times \cdots \times C P^{n_{r}}$. Let $\pi$ be the map of this multiprojective space to a point. Then $\left[M_{n}\right]=\pi_{*} c_{1}^{\Omega}\left(L_{1}^{\boldsymbol{k}_{1}} \otimes \cdots \otimes L_{r}^{\boldsymbol{k}_{r}}\right)$, where $L_{j}$ is the pull-back of the canonical line bundle on the $j$ th factor. The Chern class of this tensor product may be written using the formal group law $F^{\mathrm{a}}$ in the form $\sum \pi^{*} a_{i_{1}} \ldots i_{i} z_{1}^{i_{1}} \cdots z_{r}^{i_{r}}$, where $0 \leqq i_{j} \leqq n_{j}, 1 \leqq j \leqq r$, where $z_{i}=c_{1}^{\Omega}\left(L_{i}\right)$, and where $a_{i_{1} \cdots i_{r}} \in h(L)$. Since

$$
\pi_{*} z_{1}^{i_{1}} \cdots z_{r}^{i_{r}}=\prod_{j=1}^{r} P_{n_{j-i}}
$$

also belongs to $h(L)$, it follows that $\left[M_{n}\right] \in h(L)$. Thus $h$ is an isomorphism and the theorem is proved.

We can also give a description of the unoriented cobordism ring using formal group laws. Let $\eta^{*}(X)$ be the unoriented cobordism ring of a space $X$, that is, its generalized cohomology with values in the spectrum $M O$. There is a theory of Chern (usually called Whitney) classes for real vector bundles with $c_{i}(E) \in \eta^{i}(X)$. The first Chern class of a tensor product of line bundles gives rise to a formal group law $F^{\eta}$ over the commutative ring $\eta^{*}(p t)$. Since the square of a real line bundle is trivial, we have the identity

$$
F^{\eta}(X, X)=0
$$

THEOREM 3. The group law $F^{\eta}$ over $\eta^{*}(p t)$ is a universal formal (commutative) group law over a ring of characteristic two satisfying (6).

4. Typical group laws (after Cartier [2]). Let $F$ be a formal group law over $R$. Call a power series $f(X)$ with coefficients in $R$ and without constant term a curve in the formal group defined by the law. The set of curves forms an abelian group with addition $\left(f+{ }^{F} g\right)(X)$ $=F(f(X), g(X))$ and with operators 


$$
\begin{array}{ll}
([r] f)(X)=f(r X) & r \in R \\
\left(V_{n} f\right)(X)=f\left(X^{n}\right) & n \geqq 1 \\
\left(F_{n} f\right)(X)=\sum_{i=1}^{n}{ }^{r} f\left(\zeta_{i} X^{1 / n}\right) & n \geqq 1,
\end{array}
$$

where the $\zeta_{i}$ are the $n$th roots of 1 . The set of curves is filtered by the order of a power series and is separated and complete for the filtration.

If $R$ is an algebra over $\boldsymbol{Z}_{(p)}$, the integers localized at the prime $p$, then a curve is said to be typical if $F_{q} f=0$ for any prime $q \neq p$. If $R$ is torsion-free then it is the same to require that the series $l(f(X))$ over $R \otimes Q$ has only terms of degree a power of $p$, where $l$ is the logarithm of $F$. The group law $F$ is said to be a typical law if the curve $\gamma_{0}(X)=X$ is typical. There is a canonical change of coordinates rendering a given law typical. Indeed let $c_{F}$ be the curve

$$
c_{F}^{-1}=\sum_{(n, p)=1} \frac{\mu(n)}{n} V_{n} F_{n} \gamma_{0}
$$

where the sum as well as division by $n$ prime to $p$ is taken in the filtered group of curves and where $\mu$ is the Möbius function. Then the group law $\left(c_{F *} F\right)(X, Y)=c_{F}\left(F\left(c_{F}^{-1} X, c_{F}^{-1} Y\right)\right)$ is typical.

5. Decomposition of $\Omega_{(p)}^{*}$. For the rest of this paper $p$ is a fixed prime. Let $\Omega_{(p)}^{*}(X)=\Omega^{*}(X) \otimes \boldsymbol{Z}_{(p)}$ and let $\xi=c_{F} \Omega$. Then $\xi(Z)$ is a power series with leading term $Z$ with coefficients in $\Omega_{(p)}^{*}(p t)$, so there is a unique natural transformation $\hat{\xi}: \Omega_{(p)}^{*}(X) \rightarrow \Omega_{(p)}^{*}(X)$ which is stable, a ring homomorphism, and such that

$$
\xi_{c_{1}}^{\Omega}(L)=\xi\left(c_{1}^{\Omega}(L)\right)
$$

for all line bundles $L$.

THEOREM 4. The operation $\xi$ is homogeneous, idempotent, and its values on $\Omega_{(p)}^{*}(p t)$ are

$$
\begin{aligned}
\xi\left(P_{n}\right) & =P_{n} & & \text { if } n=p^{a}-1 \text { for some } a \geqq 0, \\
& =0 & & \text { otherwise. }
\end{aligned}
$$

Let $\Omega T^{*}(X)$ be the image of $\xi$. Then there are canonical ring isomorphisms

$$
\begin{aligned}
& \Omega \mathrm{T}^{*}(p t) \underset{\Omega_{(p)}^{*}(p t)}{\otimes} \Omega_{(p)}^{*}(X) \cong \Omega T^{*}(X), \\
& \Omega_{(p)}^{*}(p t) \underset{\Omega T^{*}(p t)}{\otimes} \Omega T^{*}(X) \cong \Omega_{(p)}^{*}(X) .
\end{aligned}
$$


$\Omega T^{*}$ is the generalized cohomology theory associated to the BrownPeterson spectrum [1] localized at $p$.

It is also possible to apply typical curves to unoriented cobordism theory where the prime involved is $p=2$. One defines similarly an idempotent operator $\xi$ whose image now is $H^{*}(X, \boldsymbol{Z} / 2 \boldsymbol{Z})$; there is also a canonical ring isomorphism

$$
\eta^{*}(p t) \otimes H^{*}(X, Z / 2 Z) \simeq \eta^{*}(X)
$$

analogous to (9).

6. Operations in $\Omega T^{*}$. If $\pi: \Omega_{(p)}^{*} \rightarrow \Omega T^{*}$ is the surjection induced by $\hat{\xi}$, then $\pi$ carries the Thom class in $\Omega_{(p)}^{*}(M U)$ into one for $\Omega T^{*}$. As a consequence $\Omega T^{*}$ has the usual machinery of characteristic classes with $c_{i}^{\Omega T}(E)=\pi c_{i}^{\Omega}(E)$ and $F^{\Omega T}=\pi F^{\Omega}$. Let $t=\left(t_{1}, t_{2}, \cdots\right)$ be an infinite sequence of indeterminates and set

$$
\phi_{t}(X)=\sum_{n \geq 0}^{F^{\Omega T}} t_{n} X^{p^{n}} \quad t_{0}=1
$$

where the superscript on the summation indicates that the sum is taken as curves in the formal group defined by $F^{\mathrm{n} T}$. There is a unique stable multiplicative operation $\left(\phi_{t}^{-1}\right)^{\wedge}: \Omega^{*}(X) \rightarrow \Omega T^{*}(X)\left[t_{1}, t_{2}, \cdots\right]$ such that

$$
\left(\phi_{t}^{-1}\right) \hat{c_{1}}(L)=\phi_{t}^{-1}\left(c_{1}^{\Omega T}(L)\right)
$$

for all line bundles $L$. This operation can be shown using (8) to kill the kernel of $\pi$ and hence it induces a stable multiplicative operation

$$
r_{t}: \Omega T^{*}(X) \rightarrow \Omega T^{*}(X)\left\lfloor t_{1}, t_{2}, \cdots\right\rfloor
$$

Writing

$$
r_{t}(x)=\sum_{\alpha} r_{\alpha}(x) t^{\alpha} \quad \text { if } x \in \Omega T^{*}(X)
$$

where the sum is taken over all sequences $\alpha=\left(\alpha_{1}, \alpha_{2}, \cdots\right)$ of natural numbers all but a finite number of which are zero, we obtain stable operations

$$
r_{\alpha}: \Omega T^{*}(X) \rightarrow \Omega T^{*}(X) .
$$

TheOREM 5. (i) $r_{\alpha}$ is a stable operation of degree $2 \sum_{i} \alpha_{i}\left(p^{i}-1\right)$. Every stable operation may be uniquely written as an infinite sum

$$
\sum_{\alpha} u_{\alpha} r_{\alpha} \quad u_{\alpha} \in \Omega T^{*}(p t)
$$

and every such sum defines a stable operation. 
(ii) If $x, y \in \Omega T^{*}(X)$, then

$$
r_{\alpha}(x y)=\sum_{\beta+\gamma=\alpha} r_{\beta}(x) r_{\gamma}(y) .
$$

(iii) The action of $r_{\alpha}$ on $\Omega T^{*}(p t)$ is given by

$$
r_{t}\left(P_{p^{n}-1}\right)=\sum_{h=0}^{n} p^{n-h} P_{p^{h}-1} t_{n-h}^{p^{h}} .
$$

(iv) If $\boldsymbol{t}^{\prime}=\left(t_{1}^{\prime}, t_{2}^{\prime}, \cdots\right)$ is another sequence of indeterminates, then the compositions $r_{\alpha} \circ r_{\beta}$ are found by comparing the coefficients of $t^{\alpha} t^{\prime \beta}$ in

$$
r_{t} \circ r_{t^{\prime}}=\sum_{\gamma} \Phi\left(t, t^{\prime}\right)^{\gamma} r_{\gamma}
$$

where $\boldsymbol{\Phi}=\left(\Phi_{1}\left(t_{1} ; t_{1}^{\prime}\right), \Phi_{2}=\left(t_{1}, t_{2} ; t_{1}^{\prime}, t_{2}^{\prime}\right), \cdots\right)$ is the sequence of polynomials with coefficients in $\Omega T^{*}(p t)$ in the variables $t_{i}$ and $t_{i}^{\prime}$ obtained by solving the equations

$$
\sum_{h=0}^{N} p^{N-h} P_{p^{h}-1} \Phi_{N-h}^{p^{h}}=\sum_{k+m+n=N} p^{m+n} P_{p^{k}-1} t_{m}^{p^{k}} t_{n}^{p^{k+m}} .
$$

This theorem gives a complete description of the algebra of operations in $\Omega T^{*}$. The situation is similar to that for $\Omega^{*}$ except the set of $\boldsymbol{Z}_{(p)}$-linear combinations of the $r_{\alpha}$ 's is not closed under composition.

\section{BIBLIOGRAPHY}

1. E. H. Brown, Jr. and F. P. Peterson, $A$ spectrum whose $Z_{p}$ cohomology is the algebra of reduced $p$-th powers, Topology 5 (1966), 149-154.

2. P. Cartier, Comptes rendus, 265 (1967), 49, 129.

3. P. E. Conner and E. E. Floyd, The relation of cobordism to $K$-theories, Lectures Notes in Math., vol. 28, 1966, Springer-Verlag, Berlin.

4. A. Fröhlich, Formal groups, Lecture Notes in Math., vol. 74, 1968, SpringerVerlag, Berlin.

5. M. Lazard, Sur les groupes de Lie formels d un paramètre, Bull. Soc. Math. France 83 (1955), 251-274.

6. S. P. Novikov, The methods of algebraic topology from the viewpoint of cobordism theories, Izv. Akad. Nauk SSSR 31 (1967), 855-951.

7. J. T. Tate, Residues of differentials on curves, Ann. Sci. Ecole Norm. Sup. 1 (1968), 149-159.

Massachusetts Institute of Technology, Cambridge, Massachusetts 02139 